\title{
以培养应用研究型人才为核心改革普通化学原理实验
}

向丹 $1^{*}{ }^{*}$, 高培红 ${ }^{1}$, 梁军艳 ${ }^{2}$, 白艳红 ${ }^{1}$, 张雯 ${ }^{2}$, 李银环 2

1 西安交通大学理学院化学实验教学中心, 西安 710049

2 西安交通大学理学院应用化学系, 西安 710049

摘要: 介绍了大类招生后, 西安交通大学化学教学实验中心针对面向理科平台学生开设的普通化学原理实验进行改 革的情况, 改革包括更新实验教学内容, 采用混合式教学方法, 融入课程思政元素和强化过程考核等, 加强对学生 的环保意识、主动探索意识、科研思维能力及实事求是的科学态度的培养及取得的效果。

关键词：普通化学原理实验; 模块化教学体系; 混合式教学方法; 课程思政

中图分类号: G64；O6

\section{Teaching Reform of the Experiment of General Chemistry Principle by Cultivating Applied Innovative Talents}

Dan Xiang ${ }^{1, *}$, Peihong Gao ${ }^{1}$, Junyan Liang ${ }^{2}$, Yanhong Bai ${ }^{1}$, Wen Zhang ${ }^{2}$, Yinhuan $\mathrm{Li}^{2}$

${ }^{1}$ Chemical Teaching Experiment Center, School of Science, Xi'an Jiaotong University, Xi'an 710049, P. R. China.

${ }^{2}$ Applied Chemistry Department, School of Science, Xi'an Jiaotong University, Xi'an 710049, P. R. China.

\begin{abstract}
This paper introduces the reform measures on the general chemistry principle laboratory, which is provided for the science-majored students by the chemistry laboratory center of Xi'an Jiaotong University after the admissions by categories. The reform involves many aspects, including reforming the laboratory teaching system, adopting the hybrid teaching method, blending in the course ideological element and strengthening the process evaluation, etc. These measures correspondingly enhanced the cultivation to the students' awareness of environmental, active exploration, scientific thinking and practical and realistic scientism. The acquired results were also presented.
\end{abstract}

Key Words: General chemistry principle experiment; Modular teaching system; Mixed teaching method; Course ideological

大类招生是高校实行 “通才教育” 的一种改革 ${ }^{[1,2]}$ 。大类招生学生入校后, 经过 1-2 年的基础 培养, 再根据兴趣爱好、专业认知自主选择专业分流。西安交通大学自 2018 年开始实行大类招生, 学校对培养计划做了调整。普通化学原理实验 ${ }^{[3]}$ (简称普化实验)作为基础科学类实验, 是针对理科 平台新生开设的 “入门级” 实验课程。课程设置体现 “厚基础、宽口径” 的教育理念, 旨在学生知 识、能力和素质的培养体系中奠定夯实的基础。

\section{1 以安全教育为切入点, 培养学生安全意识}

近几年，高校实验室安全事故多发，化工企业大火、爆炸事件频频登上各大热搜榜，“化学” 
和 “危险” 常常被一些 “标题党” 拿来放在一起博人眼球。同时, 各种原因所导致的化学污染在我 们身边随处可见。培养学生安全规范的操作习惯, 正规地处理 “三废” 应该成为每一个化学人职业 生涯的第一课。

普化实验是学生进入大学后的第一门实验课, 由于很多学生是人生中第一次进入实验室, 因此 实验室安全教育必不可少 ${ }^{4,5]}$ 。普化实验只有 32 学时, 我们抽出 1 学时专门进行安全教育, 主要从 三方面进行培训: (1) 规范实验操作, 避免因操作不当引起意外伤害。将普化实验中潜在的安全隐患 归纳、总结、提炼, 以过往事件为案例, 生动形象地向学生展示各种突发状况及其应对措施, 并将 灭火器、洗眼器等消防器材做成虚拟仿真实验, 让学生学习消防器材的使用。(2) 养成自我防护的教 育, 培养学生课前预习试剂 “特性” 的习惯。预习报告的书写必须包含实验课中涉及的所有化学品 的特性、注意事项、防护等级及防护措施等内容, 确保每一个学生对课堂上使用的化学品了如指掌。 通过自学, 学生会提前认识到课堂上会遇到的潜在危险, 提前做好防护措施。比如 “废铜制备硫酸 铜” 实验, 学生在接受安全教育后, 积极主动地用 $3 \mathrm{M}$ 口罩进行防护。学生只有对所面临的化学品 有了全面的认识，才能从心理上、技术上、方法上主动去防护。安全防护的习惯一旦养成，对他们 未来的实验安全有很大的帮助。(3) 实验教学中不断渗透环保意识, 爱护环境从 “我” 做起。破损玻 璃、废液、空试剂瓶分类投放, 同时对于四氯化碳、含铅、铬等重金属的废液单独回收。让学生从 进入实验室的第一刻起, 就养成化学品垃圾分类的好习惯。比如 “碘化铅的制备及溶度积常数的测 定”实验中，学生主动将沾有碘化铅固体的滤纸收集在一起，统一处理。

\section{2 建立模块化、内容丰富的实验教学体系}

普化实验包含 “三层次” 培养模式, 以模块化训练为主导, 力求在实验内容上体现 “趣味性” “实用性” 和 “学科交叉性”。表 1 是普化实验的具体内容及模块化训练要点。

所有实验内容都是经过多次推敲、论证, 在保障学生严格的基本训练基础上, 体现学科的交叉 性和先进性, 在不过分增加实验开发难度和成本的基础上对实验内容做出改革 ${ }^{[6]}$ 。学生带着任务去 做每一个实验, 有必须掌握的模块训练, 有拓展学生兴趣的发展空间, 有解决实际问题的实验能力, 有提前感受的先进仪器, 整个课程内容都紧密围绕培养 “强基础、重能力、有创新” 的教学理念。 实验内容要及时更新, 将科研成果转化成实验教学项目 ${ }^{[7]}$, 也是我们下一步改革的方向。

\section{3 以学生为主体, 采用混合式教学方法}

充分分析课程内容的特点及学生的接受情况, 力求课程教学效果的有效性及先进性, 在教学环 节引入混合式教学方法 ${ }^{[8,9]}$ 。

\section{1 “实验心得体会” 倒逼师生共同成长}

从第一节实验课开始, 我们就要求学生在实验结束后撰写实验心得体会。心得体会可以是成功 经验的总结也可以是失败原因的探索, 可以是对实验本身提出质疑也可以对实验室建设提出见解。 老师通过批阅实验报告, 及时了解学生的思想动态并适时做出反馈, 在师生之间建立起沟通的桥梁。 比如 “反应速率及活化能的测定” 实验, 一位学生在心得体会里书写了详细的 Excel 计算及作图的 方法, 提到 “对于 Excel 软件开始我是十分陌生的, 但经过自己的一番尝试与探索后, 掌握了一些 基本方法, 这使我认识到生活中的某些事物只是表面很棘手, 但当你真心想去了解它时, 问题会变 得显而易见! ” 还有一位同学提到, $4 \mathrm{~S}_{2} \mathrm{O}_{8}^{2-}+\mathrm{S}_{2} \mathrm{O}_{3}^{2-}+5 \mathrm{H}_{2} \mathrm{O}=10 \mathrm{SO}_{4}^{2-}+10 \mathrm{H}^{+}$应该是除了两个主反应外 的另一个副反应, 所以是不是用 $\mathrm{Na}_{2} \mathrm{~S}_{2} \mathrm{O}_{3}$ 直接去计算反应速率不太准确。在过去的几年教学中, 教 师从未仔细考虑过这个问题, 学生提出的问题倒逼教师多方查阅资料, 最终从一篇文献 ${ }^{[10]}$ 中找到答 案。一个化学反应能否发生, 要从化学热力学和化学动力学两方面进行讨论。化学热力学只解决反 应的可能性, 能否实现还需要化学动力学解决。单从热力学的角度来看, $\mathrm{S}_{2} \mathrm{O}_{8}^{2-}$ 与 $\mathrm{S}_{2} \mathrm{O}_{3}^{2-}$ 的反应不但 能发生, 而且反应进行得很完全。但此反应实际上能否发生, 还取决于反应的速度。后续我们还想 
表 1 普化实验课程体系及模块化训练要点

\begin{tabular}{|c|c|c|c|c|}
\hline 序号 & 实验类型 & 实验项目 & 模块化训练要点 & 创新点 \\
\hline 1 & 基础实验 & $\begin{array}{l}\text { 实验室安全、硫酸铜的 } \\
\text { 精制及形貌表征 }\end{array}$ & $\begin{array}{l}\text { (1) 消防器材、洗眼器等防护设备的使用、突发事件 } \\
\text { 紧急处理的应急措施; (2) 常压过滤操作; (3) 浓缩、 } \\
\text { 重结晶操作; (4) 生物显微镜的使用 }\end{array}$ & $\begin{array}{l}\text { 引入课程思政, 增加样品形貌的 } \\
\text { 表征。知识拓展: 硫酸铜晶体的 } \\
\text { 培养 }\end{array}$ \\
\hline 2 & 综合实验 & $\begin{array}{l}\text { 化学反应速率及活化能 } \\
\text { 的测定 }\end{array}$ & $\begin{array}{l}\text { (1) Excel 计算及作图; (2) 水浴锅的使用; (3) 化学 } \\
\text { 分析中条件考查的一般规律 }\end{array}$ & Excel 计算及绘图的方法训练 \\
\hline 3 & 综合实验 & $\begin{array}{l}\text { 酸碱标准溶液的配制与 } \\
\text { 标定及市售白醋中总酸 } \\
\text { 度的测定 }\end{array}$ & $\begin{array}{l}\text { (1) 分析天平的使用; (2) 容量瓶的使用; } \\
\text { (3) 酸、碱滴定管的准备、使用等; (4) 滴定操作 }\end{array}$ & $\begin{array}{l}\text { 体验式教学法, 让学生主动探索 } \\
\text { 实验结果。天然食材的引入, 增 } \\
\text { 加实验的实用性及趣味性 }\end{array}$ \\
\hline 4 & 综合实验 & $\begin{array}{l}\text { 高锰酸钾标准溶液的配 } \\
\text { 制与标定及洗涤剂中双 } \\
\text { 氧水含量的测定 }\end{array}$ & $\begin{array}{l}\text { (1) 深颜色溶液的特殊读数方法; (2) 化学稳定性不 } \\
\text { 够强的物质的配制方法; (3) 高温滴定的一般方法 }\end{array}$ & $\begin{array}{l}\text { 知识拓展: 高锰酸钾滴定泡椒风 } \\
\text { 爪中双氧水含量的应用 }\end{array}$ \\
\hline 5 & 综合实验 & 化学综合性质实验 & (1) 滴管的使用; (2) 离心机的使用 & $\begin{array}{l}\text { 翻转课堂, 让学生从 “学生” 变 } \\
\text { “老师”, 在知识的传播中加深 } \\
\text { 印象 }\end{array}$ \\
\hline 6 & 综合实验 & $\begin{array}{l}\text { 碘化铅的制备及溶度积 } \\
\text { 常数的测定 }\end{array}$ & $\begin{array}{l}\text { (1) } 721 \text { 紫外-可见分光光度计的使用; (2) Excel 计算 } \\
\text { 及作图; (3)标准曲线法 }\end{array}$ & $\begin{array}{l}\text { 知识拓展：重结晶制备黄金雨, } \\
\text { 增加实验趣味性 }\end{array}$ \\
\hline 7 & 创新实验 & $\begin{array}{l}\text { 废铁制备硫酸亚铁铵、 } \\
\text { 产品的纯度分析及形貌 } \\
\text { 检测 }\end{array}$ & (1) 比色法; (2) 热过滤操作 & $\begin{array}{l}\text { 科研素养的基本养成: 产品的合 } \\
\text { 成、表征、分析 }\end{array}$ \\
\hline 8 & 创新实验 & $\begin{array}{l}\text { 补锌口服液葡萄糖酸锌 } \\
\text { 的制备及锌含量的测定 }\end{array}$ & (1) 沉析法(不良溶剂的引入); (2) 冷冻干燥箱的使用 & $\begin{array}{l}\text { 科研思维的进一步养成: 产品的 } \\
\text { 合成、处理、分析 }\end{array}$ \\
\hline
\end{tabular}

设计一个实验去验证这个观点。学生在实验结束后的心得体会相当于对实验的复盘, 这是一个回忆、 总结、反思、提高的过程。这个习惯一旦养成, 对学生未来不管是科研还是工作都有很大的帮助, 同时也倒逼老师全面了解自己的授课环节, 取长补短、一起成长。

\section{2 体验式教学法}

滴定反应最难的部分就是对滴定终点的把握。“在酸碱标准溶液的配制与标定及食醋总酸度的 测定” 实验中, 学生非常清晰地了解甲基橙作为指示剂时滴定终点应该是黄色到橙色, 但在实际操 作中就没有那么 “自信” 了, 很多学生都依赖教师帮自己判断滴定终点。鉴于学生对终点判断的障 碍, 在过往的教学中往往采用照片、小视频等方式向学生展示滴定终点颜色的变化, 由于像素和成 像效果差, 效果通常不太理想。虽然这是一个非常成熟的实验, 但对于第一次做的学生来讲, 它就 是未知的。因此实验从设计环节开始, 就把学生主动摸索滴定终点放在了第一位, 取消了所有的结 果展示环节, 而把主动权交给学生, 让学生自己去体验 “终点” “过量” 的变化。当学生对整个滴 定过程有一个完整的体会后, 他们就能非常清晰地判断出终点。这一做法可推广到所有滴定实验中。 学生在后续的实验中能更自信地判断出滴定的终点。在保障安全的前提下, “试错” 有时候也是一 种行之有效的方法。

\section{3 翻转课堂 ${ }^{[11]}$}

以往, 大部分实验都采用教师提前讲述, 学生后续操作的流程, 但在 “化学综合性质” 实验中, 
课程开始之前教师只是对酸度计的校准、操作及离心机的使用做了详细讲述, 学生通过课前预习直 接进入实验操作的实训环节。实验结束后由小组分别对自己负责的实验部分进行讲解, 从实验现象 到反应机理, 甚至对实验中遇到的问题一一进行说明, 最后整个讨论会变成了成果分享会。学生们 都表示这种方式对知识的理解有很大的加深作用。

\section{4 信息化手段助力实验教学}

课程开课前以班级为单位建立了微信群, 群由学委和老师共同管理, 教师在课前发布下节课使 用的小型仪器的使用微视频及课程内容, 学生可以通过碎片化的时间提前完成对相关知识的学习, 也可以在群里分享实验成果、讨论实验中出现的问题, 实际课堂再由教师大致指点一下就能非常熟 练地操作仪器了。

用计算机技术进行数据处理也是重要的实验教学环节, 指导学生用 Excel 进行计算使得化学反 应速率与活化能实验的教学效果明显提高, 学生通过 Excel 计算, 大量节省了宝贵的时间和精力, 能更好地把精力放在有效数字的保留和实验结果的分析上面。

\section{4 完善考核评价体系，细化评分细则}

普化实验考核由三部分组成: 预习报告 $(20 \%)$ 、实验操作 $(40 \%)$ 、实验报告 $(30 \%+10 \%$ 心得体会)。 预习报告通过教师随机在群里点名学生回答问题来检验学生预习情况, 其他学生则通过预习报告的 书写情况给与相应分值。实验操作环节 ${ }^{[12]}$ 针对每一个实验必须掌握的技能模块进行评分, 多次出现 错误或者屡教不改者扣分。实验报告从版面的设计、数据的表达到制图, 每一项都有严格的打分标 准, 确保给出的分值公平公正。同时要向学生传递一种思想: 每一次的实验报告都是一份向别人展 示的平台，排版美观、条理清晰、数据分析有理有据是非常必要的。具体评分标准见表 2 。

\section{表 2 酸碱标准溶液的配制与标定及市售白醋中总酸度的测定实验打分标准}

\begin{tabular}{|c|c|c|}
\hline $\begin{array}{c}\text { 预习报告 } 20 \% \\
\text { 提问+检查 } \\
\end{array}$ & $\begin{array}{l}\text { 实验操作 } 40 \% \\
\text { 模块化训练要点 }\end{array}$ & $\begin{array}{r}\text { 实验报告 } 40 \% \\
30 \%+10 \% \text { 心得体会 }\end{array}$ \\
\hline 提问环节: (1) 由学生演示模块化训练要求中操作要 & (1) 分析天平的使用; (2) 容量瓶的使用; & (1) 滴定数据; (2) 数据处理 \\
\hline $\begin{array}{l}\text { 点, 教师适时纠正、补充; (2) 学生汇报试剂性质特点, } \\
\text { 根据预习情况打分 }\end{array}$ & $\begin{array}{l}\text { (3) 酸、碱滴定管的准备、使用等; (4) 滴定 } \\
\text { 操作练习 }\end{array}$ & (3) 心得体会; (4) 整体版面 \\
\hline 其他学生根据预习报告完成情况给分 & & \\
\hline (实验内容掌握情况 10 分, 模块化预习情况、试剂预习 & (每个考核点 10 分, 根据学生态度及掌握程 & (各占 10 分, 合计 40 分。) \\
\hline 情况各 5 分。合计 20 分。) & 度打分。合计 40 分。) & \\
\hline
\end{tabular}

每次实验按 100 分独立个体完成, 8 次实验最终换算成一个总成绩

\section{5 课程思政融入日常实验教学, 育才和育人两手抓}

将课程思政融入日常实验教学中, 培养学生严谨的治学态度、环保意识及爱国热情。几年前, 国产本土化妆品某品牌为了宣传自己是天然草本护肤品, 直接将 “我们恨化学” 这句不负责任的广 告词公然在各大卫视播放, 引起了不少网友的愤怒。这件事情也反映了一个问题, 化学在日常生活 中已经广泛存在了, 作为化学工作者, 我们有责任有义务在日常的教学过程中培养学生节约能源、 爱护环境, 用化学服务社会的理念。为此, 我们着重开展了三方面工作: (1) 将变废为宝的理念穿插 到实验教学环节, 旨在培养学生节物致用的理念。设计了从废铁制备硫酸亚铁铵的实验, 实验取材 自我校机械学院学生加工产生的废铁屑。从废铁屑合成出在制药、治金等方面广泛使用的硫酸亚铁 铵, 让学生充分感受到知识是变废为宝的最强力量。(2) 培养严谨的治学态度。实事求是是科研工作 的基本准则。课上明确要求实验记录时要用圆珠笔和钢笔, 严禁涂抹篡改实验数据。在 “高锰酸钾 标准溶液的配制与标定实验及天然洗涤剂中双氧水的测定” 实验中, 我们发现一位学生的数据明显 
经过多次涂改, 经过反复询问, 他承认自己由于发现和别人数据不同进行了数据改动, 后来我们一 起分析了前期高锰酸钾的称量及后续的滴定量, 最后发现他滴定的值没有问题, 但我们仍然对其任 意篡改数据的行为给予了批评, 该学生也表示以后绝不再犯。(3) 树立自信的实验心态。在做硫酸亚 铁铵的制备实验时, 有一组学生合成的溶液颜色比其他组要淡得多, 学生认为实验有误, 希望重新 做。我们询问了其具体的过程及产生的现象, 确认整个操作环节没有问题, 使他们树立自信, 最后 合成出了纯度非常高的产品。

\section{6 结语}

为了适应新形势下对人才培养的要求, 普化实验整个教学环节始终以培养学生良好的科研习惯、 严谨的治学态度、创新的科研思维为前提, 进行了系列改革, 取得了显著成效。今后, 我们会随着 课程的发展, 不断引入新的前沿课题, 只有紧跟科研发展, 不断更新实验内容和教学模式, 才能培 养出适应时代发展需要的学生。

\section{参 考 文 献}

[1] 梁明亮, 胡殿宇, 苏东民. 现代职业教育, 2017, No. 1, 16 .

[2] 高强, 李翠兰, 张晋京, 李明堂. 大学教育, 2018, No. 12, 17.

[3] 沈宏, 徐端钧, 方文军, 聂晶晶. 大学化学, 2019, 34 (4), 12.

[4] 朱莉娜, 邵松雪, 弓保津, 沈海云, 商闯. 大学化学, 2018, 33 (6), 21.

[5] 王周, 赖瑢, 彭敏, 郑赛利, 李莲云, 朱可佳, 许先芳, 陈六平, 石建新. 大学化学, 2018, 33 (10), 39.

[6] 张树永, 朱亚先, 张剑荣. 大学化学, 2018, 33(10), 1 .

[7] 向丹, 高培红, 张雯. 大学化学, 2016, 31 (2), 11.

[8] 周明娟, 张颖, 于苗, 菅文平. 大学化学, 2018, 33 (11), 67.

[9] 赖绍聪, 华洪. 中国大学教学, 2013, No. 1, 30.

[10] 李羚. 保山师专学报, 2002, 22 (2), 14.

[11] 王彦沙, 刘松艳. 大学化学, 2016, 31 (4), 17.

[12] 田福平, 张艳娟, 贺民, 贾翠英, 陈静, 孟长功. 大学化学, 2018, 33 (2), 29. 